\title{
VILA-MATAS CERCA DE VERACRUZ. CORRESPONDENCIAS (MUTUAS) CON SERGIO PITOL
}

\author{
VILA-MATAS NEAR VERACRUZ. (MUTUAL) CORRESPONDENCES \\ WITH SERGIO PITOL \\ MONIKA DĄBROWSKA \\ Universidad CEU Cardenal Herrera Valencia \\ mon_dab@yahoo.es
}

\begin{abstract}
Resumen: Enrique Vila-Matas declara a Pitol su maestro. Pocos escritores españoles actuales tienen relaciones tan cercanas con América Latina y su obra, y han sido reconocidos con diversos premios en el otro lado del Atlántico. Si bien es cierto que al autor de Lejos de Veracruz demostró con creces su proximidad literaria con varios autores hispanoamericanos, el presente artículo examina sus lazos con México a través de su amistad y convergencias literarias con Sergio Pitol. El recorrido realizado pone de manifiesto las diversas conexiones entre el autor catalán y su amigo mexicano, tanto personales, como textuales. Evidencia el papel que jugó Pitol en la configuración de la narrativa de Vila-Matas, cómo ambos se retratan mutuamente dentro de sus ficciones y el diálogo que establecen entre ellos.
\end{abstract}

Palabras Clave: Enrique Vila-Matas; Sergio Pitol; estudios transatlánticos; transtextualidad; metaliteratura

ABSTRACT: Enrique Vila-Matas declares Pitol his master. Few contemporary Spanish writers have such close relations with Latin America and its work and they have been recognized with several awards at the other side of the Atlantic. If it is true that the author of Lejos de Veracruz demonstrated his literary proximity with several Latin American authors, this article examines his ties with Mexico through his friendship and literary convergences with Sergio Pitol. Travel made through the texts highlights the various connections between the Catalan author and his Mexican friend, both personal, as the textual nature. The study evidences the role played by Pitol in the configuration of the Vila-Matas narrative, as they portray each other within their fictions and the dialogue they establish. 
Keywords: Enrique Vila-Matas; Sergio Pitol; Transatlantic Studies; Transtextuality; Metaliterature

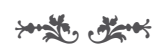

1. INTRODUCCIÓN

Fui a Xalapa como quien va a Comala. Fui a Xalapa porque me dijeron que ahí andaba quedándose a vivir Sergio Pitol, que había sido buen amigo de mi hermano Antonio. Emprendí en autocar la ruta histórica y algo extraña que une la capital de México con el puerto de Veracruz y que en el pasado sirvió de cordón umbilical entre México y España.

Enrique Vila-Matas, Lejos de Veracruz

Pocos escritores españoles actuales tienen relaciones tan cercanas con América Latina en general, y México en particular, dado el tema de este artículo, como es el caso de Enrique Vila-Matas. "Es que soy de Veracruz", se repite el protagonista de Lejos de Veracruz, el hermano del escritor Antonio Tenorio en la novela. Estas palabras, provenientes del comienzo del relato, pueden atribuirse perfectamente al propio Vila-Matas, si queremos considerar los lazos que secretamente unen su persona y su obra con la provincia mexicana, que, por otro lado, es la patria por excelencia de Sergio Pitol. Difícilmente cabe un homenaje más expresivo, el reconocimiento más elocuente de la "paternidad" literaria, de la cercanía cultural, de la admiración por las letras del país de su maestro xalapeño que las palabras antes citadas, y de paso un elogio de la escritura mexicana desde Rulfo a Pitol. Vila-Matas no solo reconoce este entrelazamiento vital entre las dos tradiciones hispánicas, sino lo explora agudamente en su obra.

A lo largo de su trayectoria, Vila-Matas atestiguó en innumerables ocasiones y de diversas maneras su estrecha conexión, tanto con la tradición literaria, como con la creación contemporánea de relevantes autores provenientes del otro lado de Atlántico. Si son muchos los autores actuales latinoamericanos que se nutren de la tradición europea y aquí encuentran reconocimiento, no abundan los prosistas españoles que hallan tanta inspiración en las letras de América Latina. Según afirma Sergio Chejfec en Una pasión hiperliteraria, "Vila-Matas está más cerca de las propuestas de escritores latinoamericanos que ningún otro escritor peninsular" (2015: en línea). Con su quehacer literario el autor de Lejos de Veracruz facilita el encuentro entre los universos narrativos concebidos por ambas orillas del mundo hispánico. Él mismo declara que se siente mejor comprendido en América que en España. De hecho, el literato catalán cuenta con una amplia proyección latinoamericana. Nunca olvida que fue reconocido primero en el otro continente, por 
críticos como Christopher Domínguez o Álvaro Enrigue, quienes en 1985 recibieron con elogios su Historia abreviada de la literatura portátil. El primer gran galardón literario lo recibió en Venezuela, el Rómulo Gallegos en 2001, seguido del Premio del Círculo de Críticos chileno dos años más tarde. Más recientemente, México lo obsequió con el Premio de Literaturas en Lenguas Romances de la Feria Internacional del Libro (FIL) de Guadalajara en 2015.

El escritor catalán ha subrayado expresa y reiteradamente su aprecio por la literatura latinoamericana y la obra de grandes maestros como Juan Carlos Onetti, Augusto Monterroso, Alejandro Rossi, Mario Levrero, José María Arguedas, Julio Cortázar, Julio Ramón Ribeyro Felisberto Hernández, Néstor Sánchez, Ricardo Piglia, Cesar Aira, Sergio Chejfec, Idea Vilariño o Julio Herrera y Reissig. Ese aprecio y diálogo lo respiran sus obras, llenas de alusiones a escritores del continente americano y sus textos. A modo de ejemplo, El mal de Montano (2002) hace referencia a Macedonio Fernández, Alejandra Pizarnik, Alan Pauls, Borges, Piglia, Aira. París no se acaba nunca (2003) está protagonizado por Monterroso, Sarduy, Borges, Cortázar, Piglia, Ribeyro, Macedonio Fernández, entre otros. En Dublinesca (2010) vuelve a evocar a Borges, Cortázar, Roberto Bolaño. Con todo, Vila-Matas es consciente de que en su país no son muchos quienes (ad)miran la creación literaria contemporánea del subcontinente. En su texto "Torre de los Panoramas" (2010), publicado en el suplemento cultural de El País, en el ciclo Café Perec, se lamenta del "duro desinterés español de ahora hacia el mundo americano", que, como dice, "perdura todavía hoy en este país obstinado en repeler ciertos registros nuevos".

La crítica ha puesto de manifiesto el denso tapiz de correspondencias, citas, diálogos intertextuales que teje Vila-Matas en su escritura con los autores de diversas geografías, tanto la española -Javier Marías, Javier Cercas, Juan José Millás- como la europea -Antonio Tabucchi, Claudio Magris, Winfried Georg Sebald (Diop 2016) - y, por supuesto, la latinoamericana (Noguerol 2016; Cintas Borrás 2012). Aquí nos detenemos en los vínculos que mantiene con Sergio Pitol, a quien se ha referido en muchos momentos en sus apuntes y memorias "Tantas veces en lugares tan distintos" (2002), "Sergio Pitol: la fuerza de la invención" (2006), "Viaje con Pitol" (2007), "Grandes lecciones de mi único maestro" (2012) y, por supuesto, en su propia narrativa.

\section{De Varsovia a Veracruz: una amistad conVertida en literatura}

Es bien sabido que a Sergio Pitol y Enrique Vila-Matas los une una larga y estrecha amistad personal. Los dos literatos han dado fe de ella en entrevistas, memorias, artículos en la prensa literaria, por no mencionar ya las numerosas fotografías donde aparecen juntos (muchas hechas durante las visitas de VilaMatas a México), reproducidas en publicaciones impresas (Domene 2002: 99; García Díaz 2007: iconografía) y en la red (por ejemplo www.enriquevilamatas. com). Pitol fue uno de los amigos escritores que mejor comprendió su obra, incluso cundo esta fue incipiente y ni siquiera conocida en España. Los dos coincidieron en Barcelona a finales de los años sesenta e inicios de los setenta, donde 
Pitol se mudó tras un breve periodo en Belgrado, pero el verdadero encuentro se produjo en Varsovia en el año 1973. Pitol ocupaba allí en aquel entonces el cargo de consejero cultural en la embajada de su país. Para el mexicano fue una segunda estadía en la capital polaca, a la que llegó por vez primera en 1963. Como escritor, acababa de publicar su primera novela, El tañido de una flauta, concebida en el periodo barcelonés. Ahora, incorporado al servicio diplomático, recibe cordialmente al joven catalán que pasa una escala obligada en Polonia camino a Egipto. Vila-Matas recuerda este momento en distintas ocasiones. En su emotivo texto "Tantas veces en lugares distintos" lo rememora así:

Te llamé por teléfono a pesar de que no nos conocíamos ni nos hablábamos y nos invitaste a almorzar y nos tomamos un vodka muy extraño que alargó mucho la sobremesa y acabamos visitando la agencia de viajes que nos alargó también la salida de Varsovia y nos quedamos en esa ciudad, invitados a tu casa, un mes entero. (Vila-Matas 2002: 55)

Esta coincidencia dio comienzo a una larga y fructífera amistad literaria. El escritor catalán pronto percibe que los encuentros culturales que Pitol organiza en su casa, las discusiones y sobremesas literarias, conversaciones en cafés, donde el mexicano empieza a fabular historias sobre la marcha, le descubren unas potencialidades artísticas hasta ahora desconocidas. "Al igual que en muchos de sus cuentos, la realidad comenzó a difuminarse", relata en el prólogo a Los mejores cuentos de Pitol (2004), otro texto muy personal y cargado de reveladores recuerdos. "Y poco a poco [las sobremesas] se llenaron de nombres que terminaron por convertirse para mí en familiares. Tolstoi, Gombrowicz, Witkiewicz, Faulkner, Henry James, Bruno Schulz...". Lo que más le sorprende al joven Vila-Matas es el interés que el mexicano muestra por su primera novela recién publicada, texto experimental e insufrible según su propia opinión, que él mismo preferiría olvidar:

Aquella mañana en Varsovia, cuando por teléfono Sergio me dijo que había leído Mujer en el espejo, me quedé de piedra y, además (lo recuerdo muy bien), me pareció el escritor mexicano un superviviente de algo, sin que acertara a saber de qué. Seguramente -me digo ahora- era el superviviente de la lectura de mi primer libro, de mi libro tremendo, del libro asfixiante, de mi verdadero primer libro asesino. [...] Ningún escritor de una generación anterior a la mía, por ejemplo, me había hablado como si yo fuera realmente un escritor. (VilaMatas 2004: 9-10)

De este primer contacto con el prosista veracruzano y los sucesos varsovianos que se prolongaron un mes entero, el visitante de Barcelona aprende una cuestión fundamental. "Una lección que venía a decirme que los personajes reales pueden llegar a convertirse en cuentos" (2004: 14). Resulta emblemática en este sentido la escena que describe en un café de Varsovia, cuando en un momento dado Sergio empieza a construir una historia a partir de lo que sucede en el local: 


\begin{abstract}
Por la tarde he recordado un café de Varsovia en el que asistí a la creación de un relato: un cuento basado en lo que mi amiga, Sergio y yo comenzamos a imaginar que sucedía en la mesa de al lado. En ella un hombre maduro, un joven que parecía su hijo, y una joven que parecía la novia del hijo tomaban aburridamente el té, pero su tedio parecía puntuado por una tensión oculta. [...] Una historia que, bajo la dirección genial de Sergio, fuimos suavemente componiendo los tres. Hasta que hubo un momento en que Sergio se disparó. Comenzó a fabular sin cesar sobre aquel trío de la mesa de al lado y parecía hacerlo como si los tres personajes fueran mexicanos, pero no estuvieran en su país, sino en un decorado con el mundo como telón de fondo. Y también parecía como si el hecho de que él los viera como mexicanos le dejara más libre para parodiar, para imaginar los diálogos, como si oír a esos personajes disparatados en su cabeza fuera para él una fuente de profunda alegría. [...] Quién sabe si nuestra amistad de tantos años no se ha fundado y refundado siempre desde la nostalgia constante de aquella tarde de risas en el café de Varsovia. (Vila-Matas 2004: 20-22)
\end{abstract}

Finalmente, Vila-Matas constata: "En aquellas fértiles sobremesas al otro lado del telón de acero se fraguó parte de lo que después, como escritor, he sido". Y añade: "La más alta lección de Sergio fue comunicarme su extraordinaria pasión por la cultura" (2004: 23). El encuentro en Polonia supuso un giro decisivo en la iniciación literaria del joven catalán, así al menos lo relata él mismo en varios lugares. El prólogo a Los mejores cuentos de Pitol es un reconocimiento de la deuda contraída al inicio de su camino y el gesto de su entrañable amistad. A partir de allí se suceden muchos encuentros con Pitol, visitas con diversos motivos y en países distintos. París, Aix-en-Provence, Madrid, Barcelona, Puerto de Santa María, Brasilia, Caracas, Mérida, Xalapa, Veracruz, Guadalajara, Morelia, México... Una constante en todos ellos son los ratos de amistad y la pasión compartida por el mundo de la escritura.

Vila-Matas no tarda en incorporar las lecciones de Varsovia a su práctica escritural. No solo escribe bajo la sombra del maestro. Da un paso más, hasta el punto de conceder a Pitol el estatus de personaje literario que una y otra vez aparece en su narrativa, personaje no solo homónimo, sino con rasgos biográficos que coinciden perfectamente con los del escritor real. Pitol no es, por supuesto, el único escritor conocido y convertido en parte de la trama en los imaginarios vilamatianos. El autor de El mal de Montano hace lo mismo con otros escritores, pero el autor xalapeño es la figura a la que alude con mayor frecuencia a lo largo de todo su ejercicio escritural. En la antología de relatos Recuerdos inventados (1994) dedica una entrada al maestro mexicano. El narrador vilamatiano se apropia en estos textos de la voz de autores que considera célebres, y uno de ellos es el amigo veracruzano. Su relato empieza así: "Me Ilamo Sergio Pitol y pienso, cuando leo a Tabucchi, en ciertos pasajes de la pintura metafísica italiana en que todo es muy nítido, muy exacto, muy certero, y al mismo tiempo definitivamente irreal".

La novela Lejos de Veracruz (1995) nace dos décadas después del encuentro en Varsovia y está definitivamente protagonizada por Sergio Pitol. El narrador-protagonista, el alter ego del autor, Ilamado Enrique Tenorio, visita a Pitol en 
la ciudad del golfo mexicano. El viaje a la provincia veracruzana se produce casi involuntariamente, cuando el narrador ya tenía billetes de regreso a España. Situación, dicho sea de paso, que evoca las circunstancias del encuentro real entre el autor de la novela y Sergio Pitol en Varsovia. En el espacio textual de Lejos de Veracruz Pitol es el amigo y el guía que ayuda a Enrique a salir de su "muerte" en términos existenciales, representada en una fatídica borrachera y crimen. Gracias a su intervención la vida del joven escritor queda conectada con el misterio de la literatura y definitivamente salvada.

El volumen Desde la ciudad nerviosa (2000) reúne las crónicas dedicadas a los intelectuales que estuvieron de paso por la ciudad libresca, Barcelona. Una de ellas, titulada "Sergio Pitol en el infierno de Escudellers", recorre la estancia pitoliana en la ciudad condal. En El mal de Montano (2002) el narrador-protagonista, un escritor bloqueado, emprende la búsqueda de su voz narrativa de la mano de Sergio Pitol y Justo Navarro. Vila-Matas confecciona en este libro el "Diccionario del tímido amor a la vida" con el fin de reunir a los diaristas que considera más importantes, y el primero de ellos es Sergio Pitol. Según justifica el narrador, "es el que lleva más tiempo colaborando en la construcción de mi tímida identidad. Personaje clave en mi vida" (2002: 192). La siguiente novela suya, si es que se le puede aplicar este término demasiado ortodoxo para la prosa vilamatiana, París no se acaba nunca (2003), relata los esfuerzos de la iniciación literaria del narrador, de la mano de Hemingway y muchos reconocidos maestros. Sergio Pitol es uno de ellos. Es el amigo del narrador y aparece en la escena donde se desvela el misterio de la antigua librería Zékian en la rue Littré:

\begin{abstract}
Hasta que una tarde, en el café de Flore, nos encontramos de pronto -no sabíamos que andaba por París y fue para nosotros una alegría-con el amigo Sergio Pitol, que se convirtió de inmediato en el jefe de la expedición al inmueble de la rue Littré. Fue él quien prácticamente nos arrastró hacia ese lugar. En cuanto aflojara la lluvia, averiguaríamos, dijo, todo lo que tuviéramos que averiguar y no nos iríamos del edificio de la calle Littré hasta que no supiéramos qué había detrás de la puerta blanca, qué clase de persona o mueble -dijo sonriendo- ocupaba el lugar exacto donde un día Borges dijo que era triste no tener recuerdos verdaderos de nuestra juventud. (Vila-Matas 2003:152)
\end{abstract}

No es de extrañar que a continuación, después de franquear la puerta, el narrador constate: "De pronto, mi mujer y yo nos miramos y, sin mediar palabra, nos entendimos de inmediato: estábamos dentro de un cuento de Pitol" (2003: 153). Este suceso es un guiño al enigmático y sombrío clima de los cuentos de Pitol, sobre todo el Ilamado Hacia Varsovia. En todos estos ejemplos el Pitol-personaje goza del estatus del incuestionable guía, preceptor y compañero de aventuras literarias.

Como es de esperar, Sergio Pitol nunca dudó en ratificar esa prolífica amistad literaria. La corrobora también dentro de su obra, introduciendo a Enrique Vila-Matas como protagonista de sus historias. La práctica de retratarse dentro de la ficción literaria es por lo tanto mutua. Ambos establecen así un tipo de juego textual, donde los dos aparecen dentro de sus respectivas ficciones 
narrativas. En El mago de Viena (2005), Pitol dedica dos capítulos a su amigo barcelonés. El primero, titulado "Vila-Matas", fue concebido con ocasión del Premio de Novela Rómulo Gallegos en Venezuela concedido al barcelonés en 2001. En su forma guarda semejanza con el diccionario de escritores imprescindibles elaborado en El mal de Montano y repasa claves y postulados esenciales de la poética vilamatiana. El mexicano, aparte de apreciación personal hacia la figura del galardonado, declara su estima por la obra que este crea:

Considero su amistad como un don extravagante y majestuoso de los dioses. [...] Lo consideraba como mi secreto hermano gemelo, mi colega de aventuras, de lecturas, de viajes, hasta que hace dos años esa relación se transformó. Con sus últimos libros, Enrique se transformó en mi maestro. (Pitol 2005: 193)

El otro capítulo de El mago de Viena, bajo el nombre "De cuando Enrique conquistó Asjabad y cómo la perdió", tiene forma de crónica de viaje a las repúblicas soviéticas de Asia. El narrador, agregado cultural en Moscú, recibe una llamada telefónica de Vila-Matas para invitarlo a participar junto con él en un festival de cine en Tashkent. Los dos acuden a la ceremonia y visitan después Asjabad en Turkmenistán. Por la noche primero cenan por equivocación en una boda, y después en un banquete con los artistas. Por una traducción tergiversada VilaMatas es tomado por un famoso cineasta y actor, sus fotografías salen al día siguiente en los periódicos. La historia recordada por el narrador es un juego entre la memoria y el ensueño. En la trama se sirve de los hechos reales para trasportarlos al terreno de la ficción, teñirlos de disparate y humor. En medio, viene un recuerdo relacionado con la persona de Enrique:

Cuando lo trataba era casi siempre con amigos cercanos, él hablaba poco, era muy introvertido, pero muy educado y agradable, eso sí. Yo había leído su primer libro Mujer en el espejo contemplando el espejo, un ejercicio de estilo como le dijo Héctor Bianciotti. Estaba entonces muy lejano de sus magníficas y excéntricas novelas ejemplares que vinieron después: Historia abreviada de la literatura portátil, Hijos sin hijos, Bartleby, una obra maestra, El mal de Montano. El Vila-Matas de Asjabad me asombraba a cada momento. (Pitol 2005: 215)

Los ejemplos traídos a colación son una clara manifestación no solo de una estrecha relación de amistad que empezó a trabarse en Polonia, sino que esta encontró un modo concreto de plasmarse en la literatura de ambos autores. Las búsquedas y la poética que elaboran el mexicano y el español a través de sus escritos conectan y se entrecruzan en muchos puntos, por eso sus caminos, personales y literarios, permanecen cercanos a lo largo del tiempo.

\section{3. ¿CÓMO HACEMOS PARA ESCRIBIR?}

Pitol y Vila-Matas comparten la preocupación por el estado de la literatura, los males que la achacan, y sobre todo el afán por renovarla. Para el veracruzano y para el barcelonés el arte de narrar y la vida "consagrada" a lo literario consti- 
tuyen un tema de fondo y un eje alrededor del cual construyen su obra. Tanto uno como otro muestran una intensa inclinación por percibir su vida a través de la vocación literaria. Aunque parezca un pleonasmo, Pitol y Vila-Matas pertenecen a esos autores para los cuales la literatura -la lectura y la escritura- son imprescindibles. Comparten la tendencia a la literaturización de la propia vida. Se podía hablar aquí de lo que Juan Carlos Onetti calificaba como literatosis, es decir una obsesión por los libros, un asociar todo lo que sucede en la vida con la literatura. "Mi vida es la literatura, vivo en la literatura", dice Pitol. "Si de algo puedo estar seguro es de que la literatura y solo la literatura ha sido el hilo que ha dado unidad a mi vida" (2005: 228). Tanto Vila-Matas, como el escritor veracruzano, llegan a corporeizar la literatura. El narrador de El mal de Montano expresa dicho estado con estas palabras: "sería conveniente y necesario [...] que me convirtiera yo en carne y hueso en la literatura misma [...] encarnarme pues en ella e intentar preservarla de su posible desaparición" (2002: 63), o en otra parte de la misma novela: "me dediqué de vez en cuando a imaginar que todo mi cuerpo [...] se transformaba por dentro y yo me convertía, me encarnaba en la memoria completa de la historia de la literatura" (2002: 310).

Pitol y Vila-Matas en muchos momentos tienen como protagonista de su discurso el propio "yo" sumergido en la actividad literaria. Esta voz está dotada a su vez de una fuerte nota de autobiografismo, que sugiere la identificación personal del escritor-personaje. Es un autobiografismo que reta la noción lejeuniana de pacto autobiográfico cfr. (Lejeune 1994). Ambos trascienden la referencialidad biográfica y la llevan al terreno de la autoficción, ${ }^{1}$ donde componen, fragmentan, re-escriben el "yo" del escritor. La metaficcionalidad -"escribo que escribo", en palabras de Barthes- es un rasgo que comparten las escrituras de ambos, y que fue reconocido por la crítica en innumerables ocasiones (Domene 2002, García Díaz 2007, Heredia 2007). Tanto la prosa pitoliana como vilamatiana procuran intencionadamente remover los límites entre lo real y lo ficticio, dejándolos permeables y confusos al situar figuras ilusoriamente reales dentro de la ficción. Con estas prácticas postulan la fusión entre ambas, la unión entre la vida y la escritura. Saben cruzar inadvertidamente la frontera entre la realidad y la ficción, construir pequeñas tramas a partir de un suceso real, ficcionalizar su propia biografía. Al referirse a la presencia de Pitol en sus primeros libros, de la década de los 90, el escritor catalán explica en un discurso en la Universidad de los Andes: "La presencia de Sergio Pitol, Fernando Pessoa y el padre de Enrique, un recio catalán nacionalista, figuras emblemáticas y paternales en todos los sentidos, acentúan el carácter falsamente autobiográfico de estas elaboradas ficciones" (Vila-Matas 2009).

Ambos escritores tematizan extensamente en las páginas de sus relatos la reflexión sobre el lenguaje y su poder de descifrar la realidad. Pitol se confiesa servidor del lenguaje, "ese don prodigioso que nos fue otorgado desde el inicio.

\footnotetext{
${ }^{1}$ La autoficción, tal y como la concibe Serge Doubrovsky con ocasion de su novela Fils (1977), hace referencia a la mezcla de lo autobiográfico con lo ficcional. La centralidad de este procedimiento en los textos pitolianos y vilamatianos la han puesto de manifiesto numerosos autores (como Pozuelo Yvancos 2010, Castro Ricalde 2000, y muchos otros).
} 
El escritor sabe que su vida está en el lenguaje, que su felicidad o su desdicha dependen de él", como dice en Mago de Viena (2005: 86). Vila-Matas también se muestra seducido por su valor críptico. En una de sus Relecturas, titulada "El fondo eterno" describe esta obsesión a través de la imagen de un sueño, protagonizado por su maestro Pitol:

Me dormí. Avanzaban en el sueño, con pasos muy presurosos, dos amigos, Sergio Pitol y Raúl Escari. Caminaban, eléctricos, por los callejones de un viejo núcleo urbano, posiblemente europeo. La lluvia, en cambio, no ofrecía dudas: era mexicana. Entraron en un aula de estudios y Sergio comenzó a escribir signos que yo nunca había visto, los escribía con gran velocidad en una pizarra de un color verde extraordinariamente potente. La pizarra se transformó en una puerta encajada en un arco ojival árabe, una puerta de un verde aún más potente y sobre la que Pitol inscribía, ralentizando el ritmo de su mano, la poesía de un álgebra desconocida: fórmulas y misteriosos mensajes de aire cabalístico, judío, aunque quizás el aire fuera sólo musulmán, musulmán de la China, o simplemente italiano, de los tiempos de Petrarca; poesía de un álgebra extraña, sin patria, que me remitía al centro del misterio del mundo. Cuando desperté, atribuí el sueño a lo que Marco Dotti me había dado a leer la noche anterior. Pero la sensación de haber estado muy cerca de un mensaje esencial-del que Pitol conocería su extensión más profunda- me fue dejando marca indeleble. (Vila-Matas 2010: en línea).

Otro punto de convergencia entre Pitol y Vila-Matas se da en el terreno de la forma narrativa. Tanto el autor mexicano como el catalán no se atienen a los límites de los géneros, los traspasan y experimentan fusionándolos. Para elaborar sus textos emplean partes de cuento, diario, relato de viaje, ensayo, fragmentos de reseñas críticas, conferencias. Como el escritor mexicano, también Vila-Matas encarna la voluntad de renovar el cuento, la novela y el ensayo, entrecruzar los géneros. "En esta fuga de géneros literarios casi todos los ensayos se imbrican con algún relato. El ensayo y la narración se unifican", dice Pitol en Una autobiografía soterrada (2011: 101). De la misma manera que Pitol en su Trilogía de la Memoria (formada por El arte de la fuga, El viaje y El mago de Viena), Vila-Matas en sus textos busca las formas de trasgredir, es decir de renovar el género.

A su vez, el carácter eminentemente híbrido y polifónico de la narrativa de ambos adquiere la forma de una condensada urdimbre de referencias, citas, evocaciones literarias, autocitaciones y fragmentos repetidos, ajenos y propios. Esta estrategia corresponde a la concepción de la escritura expresada en palabras de Justo Navarro: "Ser escritor es convertirse en un extraño, en un extranjero: tienes que empezar a traducirte a ti mismo. [...] Escribir es hacerse pasar por otro". El mal de Montano parte explícitamente de esta afirmación (2002b: 18), que vuelve en Dietario voluble (2008: 139). Pitol emplea esa misma cita en El oscuro hermano gemelo, en referencia a Vila-Matas.

La red de referencias intertextuales en los textos de Vila-Matas es extensísima, entre ellas algunas provienen de las obras de Pitol. Una muestra de ese ejercicio es la presencia del referido cuento de Pitol en Exploradores del abismo, o la inserción de las palabras provenientes de Pitol en su tomo Desde la ciudad 
nerviosa. Conviene agregar que no ocurre así a la inversa. La prosa pitoliana, marcadamente intertextual, no incorpora los textos de Vila-Matas; eso sí, evoca su figura. Las omnipresentes enumeraciones de escritores y obras leídas y comentadas, alusiones a citas, viajes y lecturas es otra práctica común en los dos autores. Dietario voluble o El mago de Viena son ejemplos elocuentes de anotaciones lectoras. De la larga lista de nombres que glosa la narrativa de ambos, se advierte el interés común por Marcel Schwob, Sebald, Kafka, Gombrowicz, Conrad, Borges, James, Rilke.

En resumen, puede concluirse la clara conciencia de Vila-Matas de encontrarse muy cerca de su amigo veracruzano, tanto en términos de una larga y comprobada amistad personal, como en cuanto a la noción de literatura, de su relación con la vida y del oficio de narrar. Desde el fortuito encuentro en Varsovia, el principiante escritor catalán encontró no solo a un compañero y un alma gemela, sino al hábil guía en la construcción de tramas que lo inició en la complejidad del arte de novelar. A partir de allí se estableció entre ambos un vínculo que Vila-Matas describe en términos de paternidad. Pitol llega a ser un maestro incuestionable. El propio Vila-Matas revindica ser discípulo suyo. Y el mexicano no duda en expresar su estima al compañero más joven. "Con sus últimos libros, Enrique se transformó en mi maestro", afirma en "El Rómulo para Enrique".

El autor catalán hace suyas y experimenta con las técnicas novelescas de Pitol. Es más, le hace aparecer en varios momentos en su obra, de manera que el autor de Veracruz se convierte en uno de los escritores más aludidos. La complicidad entre ambos se percibe tanto fuera como dentro del mundo narrativo. La afinidad es tan estrecha que llega al plano intratextual, permeando las páginas que salen de la pluma primero del escritor barcelonés, y después del propio Pitol. Los dos llevan amistad al plano literario. Con esa firme y fructífera relación, transatlántica en este caso, se inscriben en una hilera de insignes amigos literarios en la historia de las letras modernas, como lo fueron Borges y Bioy Casares, Macedonio Fernández y Borges, Rulfo y Arreola, Bolaño y Cercas, etc.).

Vila-Matas, como su maestro de Xalapa, comparten temas, escenarios, búsquedas estilísticas y estrategias narrativas comunes. Su propuesta estética se apoya sobre muchos principios comunes con la obra narrativa de Pitol. El diálogo que establece con la narrativa de Pitol a lo largo de su trayectoria como literato aumenta la sinergia entre los dos escritores. Confío en que el recorrido aquí realizado haya iluminado suficientemente el papel que jugó Sergio Pitol en la configuración del arte narrativo del autor catalán. Y viceversa, que atestigüe la íntima unión de su mundo literario con Latinoamérica, a través de Pitol. Como consecuencia del fructífero intercambio así obrado, la prosa de ambos abre nuevas brechas en la narrativa actual, en muchas direcciones, e invita a los lectores y a los teóricos a explorar los límites y las posibilidades de la literatura. Sin duda la narrativa de Vila-Matas no sería la misma sin Sergio Pitol. 


\section{OBRAS CITADAS}

Castro Ricalde, Maricruz (2000): Ficción, Narración y Polifonía: el universo narrativo de Sergio Pitol. México, Universidad Autónoma del Estado de México.

Chejfec, Sergio (2015): "Una pasión hiperliteraria", <http://www.enriquevilamatas.com/ escritores/escrchejfec8.html> [última consulta: 12.1.2018].

Cintas Borrás, Jose Vicente (2012): "Enrique Vila-Matas, Sergio Cheifec. Libros escritos por personajes de novela". En Fernández Beltrán, Francisco José y Lucía Casajús (eds.) (2012): España y América en el bicentenario de las independencias. I Foro editorial de estudios hispánicos y americanistas. Bilbao, Universidad de Deusto, pp. 515- 530.

Diop, Papa Mamour (2016): Enrique Vila-Matas en su red narrativa: hibridismo, reescritura e intertextualidad. Valladolid, Ediciones Universidad de Valladolid.

Domene, Pedro M. (2002): Monográfico "Sergio Pitol: el sueño de lo real", Batarro revista literaria, n. ${ }^{\circ}$ 38-39-40.

García Díaz, Teresa (coord.) (2007): Victorio Ferri se hizo mago en Viena. Xalapa, Universidad Veracruzana.

Heredia, Margarita (ed.) (2007). Vila-Matas portátil. Un escritor ante la crítica. Barcelona, Candaya.

Lejeune Philippe (1994): El pacto autobiográfico y otros textos [1975]. Madrid, MegazulEndymion.

Noguerol, Francisca (2016): "Lecturas casuales: Enrique Vila-Matas y sus vínculos transatlánticos", Revista Atenea, n. ${ }^{\circ}$ 514, pp. 169-188. En línea: <https://rudec.cl/index. php/atenea/article/view/52> [última consulta: 21.1.2018].

Pitol, Sergio (2001): "El Rómulo para Enrique", <http://www.enriquevilamatas. com/escritores/escrpitol1.html> [última visita: 12.1.2018].

- (2005): El mago de Viena. Valencia, Pre-Textos.

- (2011): Una autobiografía soterrada. Ampliaciones, rectificaciones y desacralizaciones. Barcelona, Anagrama.

Pozuelo Yvancos, José María (2010): Figuraciones del yo en la narrativa, Javier Marías y Enrique Vila-Matas. Valladolid, Cátedra Miguel Delibes.

Vila-Matas, Enrique (1994): Recuerdos inventados. Primera antología personal. Barcelona, Anagrama.

- (2002a): "Tantas veces en lugares tan distintos", Sergio Pitol: el sueño de lo real. Batarro revista literaria, n. ${ }^{\circ}$ 38-39-40, pp. 55-57.

- (2002b): El mal de Montano. Barcelona, Anagrama.

- (2003): París no se acaba nunca. Barcelona, Anagrama.

- (2004): Desde la ciudad nerviosa. Madrid, Alfaguara.

- (2006): "Sergio Pitol: la fuerza de la invención", Revista de la Universidad de México, n. ${ }^{\circ}$ 23, pp. 10-15. En línea: <https://goo.gl/3CeiPG> [última consulta: 14.1.2018].

— (2007): "Viaje con Pitol". En Teresa García Díaz (coord.): Victorio Ferri se hizo mago en Viena. Xalapa, Universidad Veracruzana, pp. 20-23.

- (2008): Dietario voluble. Madrid, Alfaguara.

- (2009): "Discurso en la orden del doctorado honoris causa a Vila-Matas en la Universidad de los Andes". Mérida, 10 de julio, <http://www.enriquevilamatas.com/ escritores/escrquintero1.html> [última consulta: 14.1.2018]. 
— (2010): "Torre de los Panoramas", El País. Recuperado en: <http://www.enriquevilamatas.com/textos.html> [última visita: 14.1.2018]

(2012): "Grandes lecciones de mi único maestro". En Karim Benmiloud y Raphaël Estève (eds.): Planeta Pitol. Burdeos, Presses Universitaires de Bordeaux, pp. 35-60. 